\title{
EXTENSION OF THE CAPITAL ASSET PRICING MODEL TO NON-NORMAL DEPENDENCE STRUCTURES
}

BY

MARK JOHNSTON

\begin{abstract}
The Capital Asset Pricing Model arises in an economy where agents have exponential utility functions and aggregate consumption is normally distributed, and gives the prices of assets with payoffs which are jointly normal with consumption. Such assets have normal marginal distributions and have dependence with consumption characterised by a normal copula. Wang has derived a transform which extends the CAPM by allowing pricing of assets in such an economy which have non-normal marginal distributions but still are normalcopula with consumption.

Here we set out the stochastic discount factors corresponding to this version of the CAPM and to Wang's transform, and show how to calculate stochastic discount factors and hence asset prices for assets whose dependence with consumption is non-normal. We show that the impact of dependency structure on asset prices is significant.
\end{abstract}

\section{KEYWORDS}

CAPM, stochastic discount factor, credit risk, credit spread, copula, Wang transform.

\section{INTRODUCTION}

The Capital Asset Pricing Model (CAPM), developed some forty years ago by Sharpe (1964) and Lintner (1965), is the standard model employed today to value many assets, such as companies and projects. The CAPM is typically applied as a risk-adjusted discount rate technique. The latter says that an asset with payoff $x$ (a random variable) can be valued according to the formula:

$$
p(x)=\frac{\mathrm{E}(x)}{\mathrm{E}\left(R_{x}\right)} .
$$

Here $p(x)$ denotes the price of the asset, and the number $\mathrm{E}\left(R_{x}\right)$ is the expected or required gross return on the asset. The CAPM gives a particular formula 
for the required return in terms of the relative riskiness of the asset and the market. This formula applies only to assets which satisfy the assumptions of the CAPM (e.g. assets that are jointly-normal with the market).

Contemporary equilibrium asset pricing theory is cast in terms of stochastic discount factors. These are random variables that allow assets to be priced according to formulae like:

$$
p(x)=\mathrm{E}(m x),
$$

where $m$ is the stochastic discount factor (for example see (Cochrane 2001, LeRoy and Werner 2001)). The single discount factor $m$ may be used to value all traded assets.

The CAPM can be derived under various sets of assumptions, and Cochrane (2001) gives stochastic discount factors for the CAPM for the cases of log utility and quadratic utility - but not for the case of exponential utility. The advantage of formulating the CAPM in stochastic discount factor form is that it allows one to obtain equilibrium prices for assets which are derivatives of CAPM assets, even though these derivatives do not satisfy the CAPM.

In many applications of practical importance, such as estimating the capital required to give a firm a desired degree of financial strength, one encounters some assets which could be argued to satisfy the assumptions of the CAPM - for example the physical assets of a firm - and some which are unlikely to satisfy the assumptions of the CAPM - for example the debt of the firm, which will have a payoff distribution that is far from normal. An explicit consideration of risk is also a key feature of many such applications. For example, capital requirements may be calibrated by ensuring that the probability of default on debt is less than some desired level. It is therefore important to be able to connect the valuation methods applied to these various assets, and to be able to relate valuations to explicit models of the risk of the assets.

In this paper we attempt to address these needs by developing stochastic discount factors for the CAPM and generalisations thereof, and showing how they can be applied.

Our derivation of these discount factors builds upon some papers published by Shaun Wang and Hans Bühlmann. Bühlmann (1980) developed a model for determining "optimal risk exchanges" in a closed market. Bühlmann's results can be set out in the framework of expected-utility asset pricing; specifically, his equilibrium pricing formula arises within a single-period endowment economy where agents have exponential utility functions. What we think is noteworthy about the Bühlmann paper is that it provides an explicit formula for what in contemporary terminology is the stochastic discount factor, as a function of total consumption. Wang (2003) has recently shown that the "Wang Transform," originally developed in a probability distortion operator approach to asset pricing (Wang 2002), arises also in an expected-utility equilibrium context, in a specialisation of Bühlmann's model, namely that where aggregate consumption has a normal distribution. The assets for which his transform yields equilibrium prices are those whose dependency with consumption is 
characterised by a normal copula (see e.g. (Embrechts, Lindskog, and McNeil 2001, Venter 2001) for an introduction to copulas).

Here we exhibit a formula for the marginal stochastic discount factor for these assets, which we might call the Wang assets. This discount factor is implicit in Wang's transform. Wang has pointed out that a subset of the Wang assets, those which have normally distributed payoffs, can be priced using the capital asset pricing model. Hence we have derived an explicit form for the stochastic discount factor for a common version of the CAPM, namely the version derived under assumptions of exponential utility and asset payoffs jointly normal with aggregate consumption.

We then show how to generalise the CAPM and the Wang transform and compute the marginal stochastic discount factor for any asset in this exponential-utility normal-consumption economy.

We illustrate the application of these discount factors by computing the prices (expressed as credit spreads) of debt secured over assets in this economy. We show that varying the assumed dependency structure of consumption and asset payoffs can have a significant impact on debt prices.

\section{BÜHLMANN'S ECONOMY AND WANG'S SPECIALISATION OF THIS}

In this section we will re-state Bühlmann's (1980) results in an expected-utility asset pricing framework. For a complete presentation of the relevant background material the reader may wish to refer to asset pricing texts such as LeRoy and Werner (2001), Cochrane (2001) and Panjer et. al. (1998).

In these texts it is shown that at a complete-market equilibrium in a singleperiod expected-utility endowment economy, the price $p(x)$ of an asset with payoff $x$ may be computed via the formula:

$$
p(x)=\mathrm{E}(m x),
$$

where the random variable $m$ is known as the stochastic discount factor. The equilibrium forms through agents buying and selling assets until each agent's marginal rate of substitution between consumption at the start of the period and consumption at the end of the period is the same in each state of the world. This marginal rate of substitution is the stochastic discount factor $m$. Agents' utility functions are usually formulated in terms of consumption, however in a single-period model end-of-period consumption equals end-of-period wealth (Cochrane 2001, Panjer et al. 1998). We may thus consider total consumption (denoted $c$ below) to be total wealth.

Bühlmann (1980) obtains an explicit form for the stochastic discount factor by assuming that each agent $i$ has an exponential utility function with regard to end-of-period consumption:

$$
u_{i, 1}\left(c_{i}\right)=\frac{1}{\alpha_{i}}\left(1-e^{-\alpha_{i} c_{i}}\right),
$$


where $\alpha_{i}$ is the absolute risk-aversion of agent $i$ and $c_{i}$ is this agent's end-ofperiod consumption. In this case one obtains $m$ as an exponential function of aggregate consumption:

$$
m=A e^{-\alpha c},
$$

where $c$ denotes total end-of-period consumption, $\alpha$ is the harmonic sum of the $\alpha_{i}$ :

$$
\frac{1}{\alpha}=\sum_{i=1}^{I} \frac{1}{\alpha_{i}}
$$

and $A$ is a positive constant.

Wang (2003) places a further restriction on the economy considered above, namely that aggregate consumption is normally distributed. In this economy he is able to obtain equilibrium prices for certain assets - those which are normal-copula with consumption. In a section below we will re-state his results in stochastic discount factor notation. So let us assume $c$ is normally distributed, and let $z$ be the standard normal variable obtained from $c$ by subtracting its mean and dividing by its standard deviation: $z=\left(c-\mu_{c}\right) / \sigma_{c}$. Then the discount factor can be re-written as $m=B e^{-\lambda z}$, where $\lambda=\alpha \sigma_{c}$. The stochastic discount factor thus has a log-normal distribution in this economy. Since $-\lambda z$ is a normal variable with zero mean and standard deviation $\lambda, E(m)=B e^{\lambda^{2} / 2}$, and if a risk-free asset is traded, so that $E(m)=1 / R_{f}$ where $R_{f}$ is the risk-free gross return (see e.g. (Cochrane 2001)), the constant $B$ is thus $e^{-\lambda^{2} / 2} / R_{f}$, i.e.

$$
m=\frac{1}{R_{f}} e^{\frac{-\lambda^{2}}{2}} e^{-\lambda z} .
$$

Equation (1) gives the stochastic discount factor in this economy. In the following section we will show how to graph the stochastic discount factor as a function of the payoff of any particular asset.

\section{MARGINAL DisCOUNT FACTORS}

Suppose $x$ is an asset, $m$ is a stochastic discount factor, and suppose that $m$ and $x$ are jointly continuous with density $f_{m x}$. Consider a derivative $y$ of $x$, meaning an asset with payoff of the form $y=g(x)$ for some function $g$. We call it a derivative of $x$ because its payoff is determined by the payoff of $x-$ an example is a call option over a stock. Writing $f_{x}$ for the probability density function of the random variable $x$, the value of this derivative is given by:

$$
\begin{aligned}
E(m y) & =\int_{s \in \mathbf{R}} \int_{r \in \mathbf{R}} r g(s) f_{m x}(r, s) d r d s \\
& =\int_{s \in \mathbf{R}} g(s) f_{x}(s)\left(\int_{r \in \mathbf{R}} r \frac{f_{m x}(r, s)}{f_{x}(s)} d r\right) d s
\end{aligned}
$$




$$
\begin{aligned}
& =\int_{s \in \mathbf{R}} m_{x}(s) g(s) f_{x}(s) d s \\
& =E\left(m_{x} g(x)\right),
\end{aligned}
$$

where $m_{x}(s)$ is the quantity in brackets. The quantity $f_{m x}(r, s) / f_{x}(s)$ is the conditional density with respect to $x$. Thus $m_{x}$ is the conditional expectation of the SDF with respect to $x$.

As $m_{x}$ is defined in the same way as the marginal probability density of $x$, that is by integrating over the level sets of $x$, we refer to $m_{x}$ as the marginal discount factor of $x$. Since the marginal density is all one needs to calculate expectations of functions of $x$, the marginal density and marginal discount factor are all one needs to compute the values of functions ("derivatives") of $x$.

It is not necessary to assume $m$ and $x$ are jointly continuous - if $m$ is measurable and $y$ is a derivative of $x, y=g(x)$, where $g$ is a Borel-measurable function on the real line, then as long as $m y$ is measurable, we have:

$$
E(m y)=E(E(m y \mid x))=E(y E(m \mid x)),
$$

where the first equality is the law of iterated expectations and the second uses the fact that if $g$ is measurable on $\mathbf{R}$ then $y$ is measurable on the sigma-algebra generated by $x$.

\section{MARginAl Discount FACTOR FOR WANG ASSETS}

What Wang (2003) effectively derives is a marginal stochastic discount factor for any asset $x$ which is normal-copula with consumption.

Assume an asset $x$ is normal-copula with total consumption, meaning here that $x$ may be written as $x=h(v)$, where $h$ is an increasing function, and $v$ and $z$ have a standard bi-variate normal distribution with correlation coefficient $\rho_{c v}$. The function $h(v)$ is $F_{x}^{-1}(\Phi(v))$, where $F_{x}$ is the cumulative distribution function (CDF) of $x$ and $\Phi$ is the CDF of a standard normal variable.

Consider a derivative of $x, y=g(x)$. Following Wang we can show that the value of any such derivative is given via the one-dimensional integral:

$$
p(y)=E\left(m_{x} y\right)=\int_{-\infty}^{\infty} m_{x}(s) g(s) f_{x}(s) d s,
$$

where $f_{x}$ is the marginal probability density of $x$, and

$$
m_{x}(s)=C e^{-\lambda \rho_{c v} h^{-1}(s)},
$$

and $C$ is a constant. The function $m_{x}$ is the marginal discount factor for $x$, as per our definition above.

The fact that the variable $v$ is jointly-normal with consumption means that all information about the dependence between $v$ and consumption is captured 
by a single number, the correlation coefficient. In general the marginal stochastic discount factor for an asset will contain whatever parameters are needed to describe the copula between consumption and the asset.

When a risk-free asset is traded, the constant $C$ above may be determined by requiring that the expected value of $m_{x}$ be $1 / R_{f}$, in which case we find that:

$$
m_{x}(s)=\frac{1}{R_{f}} e^{-\frac{\lambda_{x}^{2}}{2}} e^{-\lambda_{x} h^{-1}(s)},
$$

where the parameter $\lambda_{x}=\lambda \rho_{c v}$.

\subsection{Connection with the Wang Transform}

In the remainder of the paper (Wang 2003), Wang goes on to show that the prices of Wang assets may also be calculated as:

$$
p(x)=\frac{1}{R_{f}} E^{Q}(x),
$$

where $E^{Q}$ represents the expectation with respect to a transformed probability distribution $F_{x}^{Q}$, the Wang Transform of $F_{x}$, defined by

$$
F_{x}^{Q}(s)=\Phi\left(\Phi^{-1}\left(F_{x}(s)\right)+\lambda_{x}\right) .
$$

A distribution which allows pricing via an equation such as (3) is usually known as a risk-neutral probability distribution (for example see (Cochrane 2001, LeRoy and Werner 2001)), so in this equilibrium pricing context the Wang transform turns the "real-world" or "physical" probability distribution of an asset into its risk-neutral distribution.

Note that by differentiating Equation (4) we can establish a formula for the risk-neutral probability density function:

$$
f_{x}^{Q}(s)=\left(F_{x}^{Q}\right)^{\prime}(s)=e^{-\frac{\lambda_{x}^{2}}{2}} f_{x}(s) e^{-\lambda_{x} \Phi^{-1}\left(F_{x}(s)\right)},
$$

where $f_{x}(s)$ is the original probability density function: $f_{x}(s)=F_{x}^{\prime}(s)$.

We may then use the fact that the stochastic discount factor, scaled by its mean, is the "change of measure" which relates the risk-neutral probabilities to the physical probabilities:

$$
f^{Q}(s)=f(s) \frac{m(s)}{E(m)},
$$

to recover the formula (2) for the stochastic discount factor $\left(\right.$ where $h^{-1}(s)=$ $\left.\Phi^{-1}\left(F_{x}(s)\right)\right)$.

A connection may also be made with the methods of distortion and relative entropy minimisation - one may show that equation (2), with $\lambda_{x}$ considered 
a parameter, defines an exponential family of distortions of the original probability density function (see (McLeish and Reesor 2003)), and that the marginal discount factor (scaled by its mean) is the distortion which solves the minimum relative entropy problem with constraint $E^{Q}(v)=-\lambda_{x}$ (i.e. the constraint is that it correctly prices the underlying normal asset).

\section{STOCHASTIC DISCOUNT FACTOR FOR CAPM ASSETS}

We have shown that in a single-period endowment economy, where agents are expected-utility maximisers, with the end-of-period utility being exponential, and where the total end-of-period endowment/consumption is normally distributed, we can derive an explicit expression for the stochastic discount factor in terms of total consumption, and that for certain assets within this economy, namely those which are normal-copula with consumption, we can derive an explicit expression for the marginal stochastic discount factor.

Suppose we have an asset $x$ which is normal-copula with consumption and has a payoff which is normal with mean $\mu_{x}$ and standard deviation $\sigma_{x}$ (so it is jointly normal with consumption). Then it may be related to a unit normal variable $v$ via the increasing transformation $x=h(v)=\mu_{x}+\sigma_{x} v$. The inverse transformation is $h^{-1}(x)=\left(x-\mu_{x}\right) / \sigma_{x}$, so we see from (2) that the marginal stochastic discount factor for $x$ is:

$$
m_{x}(s)=\frac{1}{R_{f}} e^{-\frac{\lambda_{x}^{2}}{2}} e^{-\lambda_{x} \frac{\left(s-\mu_{x}\right)}{\sigma_{x}}},
$$

so the marginal stochastic discount factor for such an asset is exponential in the payoff of the asset.

The reader may recall that assumptions of exponential utility and asset payoffs jointly normal with consumption give one way of deriving the Capital Asset Pricing Model (CAPM) (see e.g. (Cochrane 2001)). We thus conclude that Equation (6) gives the marginal stochastic discount factor for an asset which satisifies this version of the CAPM. We will call the assets which are jointly normal with consumption the CAPM assets.

If $x$ and $y$ are jointly normal with consumption, then so is $a x+b y$ for any real numbers $a$ and $b$, so the space of CAPM assets is closed under linear combinations, and so forms a vector subspace of the space of all assets. This means that the CAPM assets are somewhat special in that a portfolio of CAPM assets is a CAPM asset. This property does not hold for the Wang assets, for example - copulas are not guaranteed to be preserved under addition.

\subsection{Pricing with the CAPM discount factor}

We can obtain analytical formulae for the values of CAPM assets and certain derivatives of them, as we will now illustrate. In general numerical integration is necessary to obtain asset values. 
Suppose $x$ is a derivative of a CAPM asset $v: x=g(v)$. Without loss of generality we can assume $v$ is unit normal (a derivative of a CAPM asset is also a derivative of a unit CAPM asset). Then we may value $x$ using the marginal discount factor of $v$ as follows:

$$
\begin{aligned}
p(x) & =E\left(m_{v} g(v)\right) \\
& =\int_{-\infty}^{\infty} m_{v}(s) g(s) f_{v}(s) d s \\
& =\int_{-\infty}^{\infty} \frac{1}{R_{f}} g(s) e^{-\frac{\lambda_{v}^{2}}{2}} e^{-\lambda_{v} s} e^{\frac{-s^{2}}{2}} \frac{1}{\sqrt{2 \pi}} d s \\
& =\int_{-\infty}^{\infty} \frac{1}{R_{f}} g(s) \frac{e^{-\frac{\left(s+\lambda_{v}\right)^{2}}{2}}}{\sqrt{2 \pi}} d s \\
& =\int_{-\infty}^{\infty} \frac{1}{R_{f}} g\left(s-\lambda_{v}\right) \frac{e^{\frac{-s^{2}}{2}}}{\sqrt{2 \pi}} d s \\
& =\frac{1}{R_{f}} E\left(g\left(v-\lambda_{v}\right)\right)
\end{aligned}
$$

This is a convenient formula for pricing normal assets and their derivatives. We will consider a couple of examples below. Note that the $\lambda$ used is that of $v$, i.e. $\lambda_{v}=\rho_{c v} \lambda$. This pricing formula is basically an implementation of Wang's conclusion that the risk-neutral distribution for a CAPM asset $x$ is just the physical distribution shifted to the left by $\lambda_{x} \sigma_{x}$ (Wang 2002). Any asset which is normal-copula with consumption is a derivative of a CAPM asset.

Value of a CAPM asset. Let $x$ be a CAPM asset with mean $\mu$ and standard deviation $\sigma$. Then $x$ is a derivative of a unit CAPM asset $v$ via $x=g(v)=\mu+\sigma v$, so the price of $x$ is:

$$
p(x)=\frac{1}{R_{f}} E\left(\mu+\sigma\left(v-\lambda_{v}\right)\right)=\frac{1}{R_{f}}\left(\left(\mu-\lambda_{v} \sigma\right)+\sigma E(v)\right)=\frac{1}{R_{f}}\left(\mu-\lambda_{v} \sigma\right) .
$$

As $x$ is a linear function of $v$, it has the same correlation with total consumption, so $\lambda_{v}=\rho_{c v} \lambda=\rho_{c x} \lambda=\lambda_{x}$.

Value of an asset whose log satisfies the CAPM. Let $x=g(v)=e^{\mu+\sigma v}$, where $v$ is a unit CAPM asset. Then the price of $x$ is:

$$
p(x)=\frac{1}{R_{f}} E\left(e^{\mu+\sigma\left(v-\lambda_{v}\right)}\right)=\frac{1}{R_{f}} e^{\mu-\lambda_{v} \sigma} E\left(e^{\sigma v}\right)=\frac{1}{R_{f}} e^{\mu-\lambda_{v} \sigma+\sigma^{2} / 2} .
$$

The reader may verify that a the price of a call option on such an asset is given by the Black-Scholes formula (Black and Scholes 1973, Black and Scholes 1972), as shown by Wang (2002). 
Value of a call option on a CAPM asset. Let $x=\max (0, y-K)$, where $y$ is a CAPM asset: $y=\mu+\sigma v$. Then $x=\max (0, \mu+\sigma v-K)$, so the price of $x$ is:

$$
p(x)=\frac{1}{R_{f}} E\left(\max \left(0, \mu-K+\sigma\left(v-\lambda_{v}\right)\right)\right)=\frac{\sigma}{R_{f}} E(\max (0, a+v)),
$$

where $a=\left(\mu-\lambda_{v} \sigma-K\right) / \sigma=\left(R_{f} p(y)-K\right) / \sigma$ is a "distance in-the-money". Hence

$$
p(x)=\frac{\sigma}{R_{f}} \int_{-a}^{\infty}(a+s) \phi(s) d s=\frac{\sigma}{R_{f}}(a \Phi(a)+\phi(a)),
$$

where $\phi$ and $\Phi$ are respectively the probability density function and cumulative distribution function of a standard normal variable.

Using put-call parity we can obtain a formula for the price of the corresponding put option, which we will use in the debt pricing examples given later in this paper.

\subsection{Meaning of the parameter $\lambda_{x}$}

For any asset $x$ with non-zero price the Sharpe Ratio is defined as the ratio of expected excess return to standard deviation of return (see (Cochrane 2001)):

$$
\theta_{x}=\frac{E\left(R_{x}\right)-R_{f}}{\sigma_{R_{x}}} .
$$

This ratio is also known as the market price of risk of asset $x$, as it shows how much excess return will be demanded per unit of risk (measured as return standard deviation).

One can show that for any asset $x$ we have:

$$
\theta_{x}=\rho_{m x} \theta_{m}
$$

In words: the Sharpe Ratio for an asset $x$ is the product of the Sharpe Ratio of the stochastic discount factor and the linear correlation coefficient of the asset with the discount factor.

Suppose $x$ is a CAPM asset with parameters $\mu_{x}, \sigma_{x}, \lambda_{x}$. Then the gross return on $x, R_{x}=x / p(x)$, is also a CAPM asset, with mean $E\left(R_{x}\right)=\mu_{x} / p(x)$ and standard deviation $\sigma_{R_{x}}=\sigma_{x} / p(x)$. Rearranging Equation (7) shows that for CAPM assets the parameter $\lambda_{x}$ is the Sharpe ratio of $x$ :

$$
\lambda_{x}=\frac{\mu_{x}-R_{f} p(x)}{\sigma_{x}}=\frac{E\left(R_{x}\right)-R_{f}}{\sigma_{R_{x}}} .
$$

Since total consumption, $c$, is normal in the economy we are considering, and has $\lambda_{c}=\lambda$, we may interpret $\lambda$ as the Sharpe ratio of consumption, and refer to it as the overall market price of risk. 
From the relationship $\lambda_{x}=\rho_{c x} \lambda$ one can then derive the usual formulation of the CAPM in terms of "beta" (see e.g. (Panjer et al. 1998)). As pointed out earlier, in a single-period model total end-of-period consumption equals total end-of-period wealth, so the return on consumption $R_{c}$ may be thought of as the return on wealth, or the return on "the wealth portfolio", which is how the CAPM is usually presented.

For assets $x$ which do not satisfy the CAPM (e.g. an asset which is normalcopula with consumption but has a Gamma marginal distribution, or an asset which is Clayton-copula with consumption) the parameter $\lambda_{x}$ will not in general equal the Sharpe ratio $\theta_{x}$.

From the relationship $\lambda_{x}=\rho_{c x} \lambda$, and the fact the correlation coefficients are no larger than one in absolute value, one reaches the classical result that normal assets can have a Sharpe ratio no greater than that of the wealth portfolio so the wealth portfolio is efficient among normal assets in the sense that it offers the greatest ratio of excess return to risk. Other assets may yield higher Sharpe ratios, however. Referring to Equation (10) we see that the upper bound on the absolute value of the Sharpe ratio of an asset is the absolute value of the Sharpe ratio of the discount factor. The Sharpe ratio of the discount factor is proportional to the standard deviation of the discount factor: $\theta_{m}=-R_{f} \sigma_{m}$ (see e.g. (Cochrane 2001)). In the present case the latter may be computed from Equation (1) and the formula for the standard deviation of a log-normal distribution. In this manner we arrive at the following formula for the Sharpe ratio of the discount factor in this economy:

$$
\theta_{m}=-\sqrt{e^{\lambda^{2}}-1}=-\left(\lambda+\frac{\lambda^{3}}{4}+\frac{5 \lambda^{5}}{96}+\ldots\right)
$$

We see that the Sharpe ratio of the discount factor exceeds that of consumption in absolute value. Thus one could obtain a Sharpe ratio exceeding that of the wealth portfolio by shorting the discount factor, for example. While the wealth portfolio is efficient among normal assets, it is thus not efficient in the space of all assets. When $\lambda$ becomes large, the maximum Sharpe ratio implied by Equation (11) will be quite a bit higher than that of consumption, $\lambda$. Accordingly if we think of our single-period model being applied to a reasonably long time period, over which quite large Sharpe ratios can be obtained by investing in equities, for example, the value of $\lambda$ required to accommodate these would be significantly lower than that required if we consider normal assets only.

The possibility of achieving Sharpe Ratios higher than those of the wealth portfolio is not present in the other versions of CAPM presented in Cochrane (2001) (log utility and quadratic utility). In those models the discount factor is linear in the wealth portfolio return, meaning it is of the form $m=a+b R_{W}$, where $R_{W}$ is the return on the wealth portfolio (which is aggregate consumption in a one-period model) and $a$ and $b$ are constants. The Sharpe Ratio of the discount factor is then obviously that of the wealth portfolio, so the latter serves as an upper bound on the Sharpe Ratios of all assets. 


\section{Pricing Debt SeCured over CAPM Assets}

Before going on to generalise the above results, let us consider how to price derivatives of CAPM assets using the CAPM stochastic discount factor. The example we will consider is zero-coupon debt secured over assets which satisfy the CAPM. The debt is then valued as a derivative of the assets, in the style of the original models of Black and Scholes (1973) and Merton (1974). Whereas in Merton's approach one works from an observed volatility and price of equity to the volatility of the underlying assets and thence to a price for the debt, here we will assume the price and distribution of the payoff of the underlying assets is known, and compute the price of the debt. The example is intended to serve as an illustration of how to use and interpret the marginal discount factor, and to provide a reference case for the subsequent examples involving other copulas.

To work out this and subsequent examples we will need to make some assumptions about global parameters such as the risk-free rate and market price of risk. We will suppose the debt has a two-year maturity and take the risk-free return over the two-year period to be $R_{f}=1.1$, and the market price of risk over the two-year period to be $\lambda=0.6 \sqrt{2} \simeq 0.85$. These are chosen to correspond roughly with what one might currently observe in real markets, being a risk-free rate of $5 \%$ per annum, and a market Sharpe ratio which is 0.6 over one year and grows initially with the square root of time.

Let us assume the assets of the firm are normally distributed, with the payoff in two years having a mean of 100 and a standard deviation of 20 , and furthermore are jointly-normal with consumption with correlation 0.5 . These are the three asset-specific parameters we need to value the assets. We can compute the assets' price of risk as $0.5 * \lambda=0.42$, and apply the valuation formula for a CAPM asset (Equation (7)), to get the asset value $A_{0}=83$ (we are giving everything to two significant figures only in these examples). We'll denote payoffs at maturity (time $T$ ) by a subscript $T$, and the values (at time zero) of these payoffs by a subscript 0 .

Let us suppose the face value of the debt is $C_{T}=64^{1}$. If the payment of this face value was guaranteed, the value of the debt would be $C_{0}=C_{T} / R_{f}=58$. However, debt holders will receive the face value at maturity only if the assets are sufficient to cover it, and otherwise they will receive the payoff of the assets $^{2}$. The payoff to debt holders is thus: $L_{T}=\min \left(C_{T}, A_{T}\right)^{3}$. To value the debt we just need to integrate this payoff against the physical probability distribution

1 You should in fact use $C_{T}=63.86$ if you want to replicate our answers - in working out the example we solved for $C_{T}$ to give the desired credit spread.

2 Real market features not considered here, such as taxes, bankruptcy costs, and variations from absolute priority of creditors, could however be incorporated, as illustrated for this class of model by Leland (1994, 1996).

3 Note that since the assets are assumed to be normally distributed there is the possibility of the asset payoff, and hence this "debt" payoff, being negative. We shall ignore this in this paper, as for the parameter values used the value impact of the negative part of the payoff will be negligible. 
of the assets and the marginal stochastic discount factor for the assets. The resulting debt value is $L_{0}=57$. The relationship between the promised payoff of $C_{T}=64$ and the debt value $L_{0}=57$ can be expressed as a credit spread, and here this is 60 basis points ${ }^{4}$, which corresponds to quite highly-rated debt around AA for a two-year maturity (Amato and Remolona 2003). The valuation process is illustrated graphically in Figure 1.

One can see how the valuation process illustrated here would apply to any derivative of a CAPM asset. In the case of debt, however, it is instructive to decompose the payoff of the debt into a promised amount (the "claim") of $C_{T}$, less a put option on the assets with strike $C_{T}$, as in the original models of Black and Scholes and of Merton. Let us denote the payoff of the put option by $D_{T}=\max \left(0, C_{T}-A_{T}\right)$. We will refer to it as the default loss or just the loss. Terms such as "expected loss" will then have the meaning they do in the field of credit risk (see e.g. (Schonbucher 2003)). The payoff of the debt is $L_{T}=$ $C_{T}-D_{T}$, and the value of the debt is hence the value of the claim less the value of the default loss: $L_{0}=C_{0}-D_{0}$. The first term will be constant across all of our examples, being determined by the face value of the debt and the risk free rate - it is the second term which varies with the risk of the assets. This decomposition allows one to more clearly isolate the effect of the marginal discount factor, as illustrated in Figure 2.

We see that since the correlation between the assets and consumption was taken to be positive the marginal discount factor places greater weight on those states of the world where the asset has a low payoff, relative to those where it has a high payoff (the marginal SDF slopes downwards). In particular, those states of the world where the company defaults (where the loss is non-zero) receive greater weights. This raises the risk-neutral default probability relative to the physical default probability. This "risk premium" effect is considered to be a significant component of observed debt prices (Amato and Remolona 2003).

If the correlation was taken as negative the marginal SDF would slope upwards, and if the correlation was zero the marginal SDF would be horizontal, taking the value $1 / R_{f}$ regardless of the payoff of the assets.

As is obvious from this discussion and from consideration of Figure 2, for a given asset payoff distribution, and hence given default probability, the pricing of debt will vary with the correlation of the underlying asset with consumption, as the latter parameter determines the slope of the CAPM marginal discount factor, and hence the weights used in valuing the default loss. We note however that if a different copula is assumed then the relationship between asset correlation and debt prices will be different, as we shall see later.

4 For a zero-coupon bond of maturity $T$, face value $C_{T}$ and market value $L_{0}$, the credit spread is the number $c$ satisfying: $L_{0}=e^{-\left(r_{f}+c\right) T} C_{T}$, where $r_{f}$ is the continuously-compounding risk-free rate for maturity $T\left(R_{f}=e^{r_{f} T}\right)$. Solving for the spread gives: $c=\frac{1}{T} \ln \left(\frac{C_{0}}{L_{0}}\right)$, and we can take this as the definition of the credit spread even in cases where the claim amount $C_{T}$ is uncertain. 

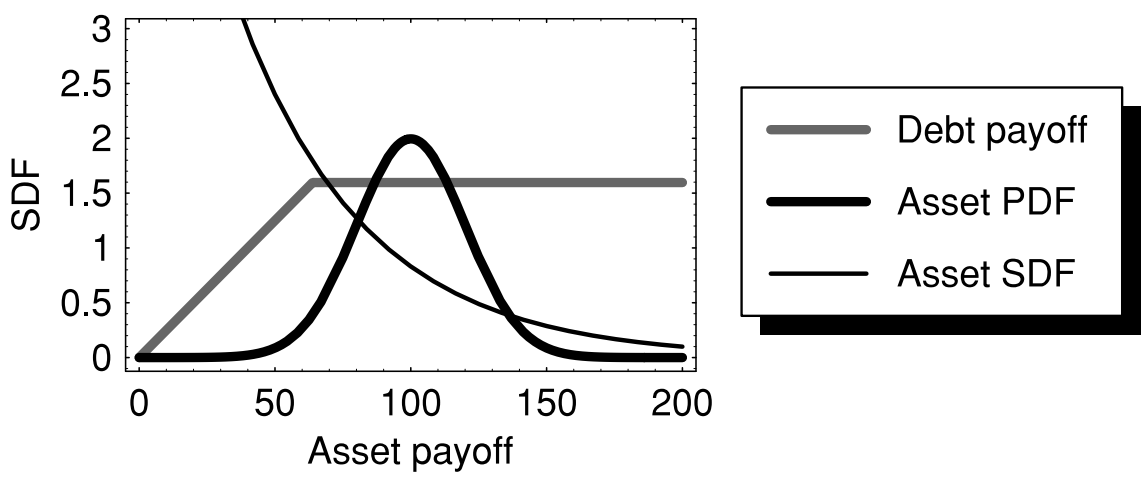

FIGURE 1: Elements necessary to value debt: the debt payoff as a function of the asset payoff, the probability density function of the assets, and the marginal stochastic discount factor of the assets. To calculate the value of the debt we multiply the debt payoff by the asset SDF before integrating against the asset PDF. As the assets satisfy the CAPM this integral can be computed analytically (Equation (8)).

The units on the vertical axis are those of the SDF; the other graphs have been scaled to fit.

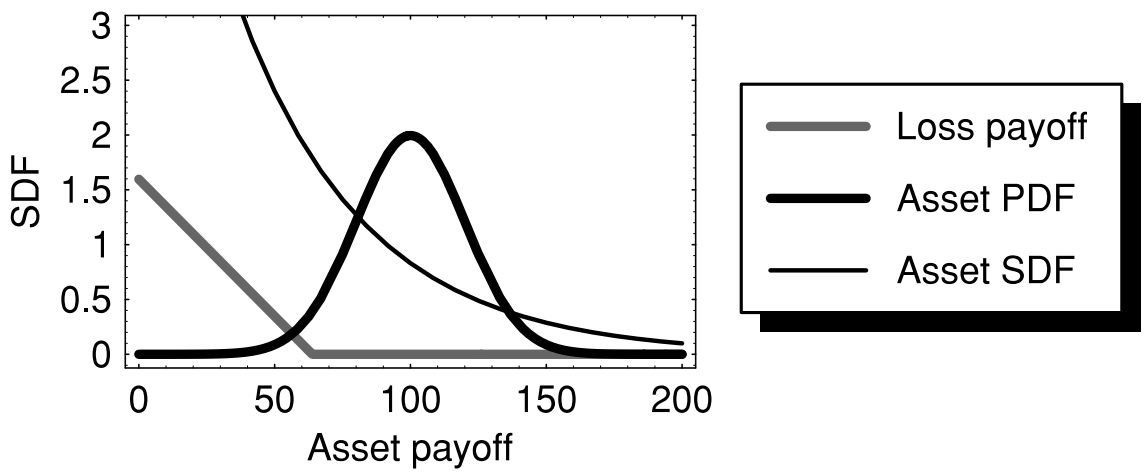

FIGURE 2: The effect of the asset SDF on debt value can be seen more clearly through its effect on the default loss. To calculate the expected loss we simply integrate the loss payoff against the asset PDF.

To calculate the value of the loss we multiply the loss payoff by the asset SDF before integrating against the asset PDF. As the SDF has values greater than $1 / R_{f}$ where the loss payoff takes non-zero values, the value of the loss will exceed the present value of the expected loss.

\section{STOCHASTIC DisCOUNT FACTOR WHEN THE COPULA IS NOT NORMAL}

Above we have shown how to obtain an analytical formula for the marginal SDF for assets which have arbitrary marginal distribution but are normalcopula with consumption. In this section we will show how to compute the marginal stochastic discount factor for assets whose dependency with consumption is characterised by some other copula.

Recall that in the economy we are considering, the stochastic discount factor is log-normally distributed, with the form shown in Equation (1). Since the 
stochastic discount factor is a function of the scaled consumption $z$, we may write the marginal SDF of any asset $x$ as an integral over $z$ :

$$
m_{x}(s)=\int_{-\infty}^{\infty} m(z) \frac{f_{z x}(z, s)}{f_{x}(s)} d z,
$$

where $f_{x}$ is the marginal density of $x$ and $f_{z x}$ is the joint density of $z$ and $x$. Suppose we know the distribution of $x$. Since we know the distribution of $z$, the additional element we need in order to obtain the marginal discount factor for $x$ is the copula of $z$ and $x$. Since consumption is a strictly increasing function of $z$, and copulae are invariant under strictly increasing transformations of variables (see e.g. (Embrechts, Lindskog, and McNeil 2001)), we can refer to this as the copula of consumption and the asset $x$. The joint density of $z$ and $x$ may be separated into a product of the marginal densities and the copula density (see e.g. (Embrechts, Lindskog, and McNeil 2001)), which we will write as $c_{z x}$ :

$$
f_{z x}(r, s)=\phi(r) f_{x}(s) c_{z x}\left(\Phi(r), F_{x}(s)\right)
$$

(here $F_{x}$ and $f_{x}$ respectively denote the cumulative probability function and the probability density of $x$ ). The marginal SDF then becomes:

$$
m_{x}(s)=\frac{e^{\frac{-\lambda^{2}}{2}}}{R_{f}} \int_{-\infty}^{\infty} e^{-\lambda z} c_{z x}\left(\Phi(z), F_{x}(s)\right) \phi(z) d z
$$

Completing the square yields the following formula for the marginal SDF of an asset $x$ in this economy:

$$
m_{x}(s)=\frac{1}{R_{f}} \int_{-\infty}^{\infty} c_{z x}\left(\Phi(z-\lambda), F_{x}(s)\right) \phi(z) d z .
$$

Equation (12) allows us to price any asset in this economy as long as we know the marginal distribution of the asset and are prepared to speculate upon its copula with consumption. In general the computation of the marginal SDF at a point will require numerical evaluation of a one-dimensional integral. The valuation of an asset will thus require numerical evaluation of a two-dimensional integral. One can of course verify that in the case where the asset's marginal distribution is normal and the normal copula is assumed, we can analytically evaluate the integral and recover the CAPM stochastic discount factor derived earlier (Equation (6)). Equation (12) is thus a generalisation of the CAPM.

\subsection{Examples: Student-t and Clayton copulas}

To isolate the impact of changing the assumed dependency structure only, we will consider here the CAPM example given above, and work out the impact 
on credit spread when the assets are assumed to have a normal marginal distribution and be Student-t-copula with consumption, or Clayton-copula with consumption.

We choose the Student-t copula (see (Demarta and McNeil 2004)) as it is a natural generalisation of the normal copula, but one which exhibits the property of tail dependence. In a bi-variate normal distribution the likelihood of one variate being below its $\alpha$-quantile given that the other is below its $\alpha$-quantile approaches zero as $\alpha$ is lowered towards zero, whereas with a Student-t copula this likelihood is non-zero in the limit. In the present context this amounts to a higher likelihood of aggregate consumption and the asset payoff being simultaneously very low. Accordingly we will see a higher risk premium being demanded for assuming the low end of the asset's payoff, as one does when one takes on debt written over the asset.

Samples from a multivariate Student-t distribution with $v$ degrees of freedom can be generated by generating correlated variate samples from a multivariate normal distribution and dividing these by a factor drawn from a chi distribution with $v$ degrees of freedom (Demarta and McNeil 2004). One could think of the chi distribution as providing systematic shocks, impacting all variates at once. A bivariate Student-t copula thus has two parameters - one being the degrees of freedom parameter $v$ and the other being the correlation between the underlying normal variates. For the sake of producing an example we will arbitrarily set the degrees of freedom parameter to 5 , but to facilitate a fair comparison we will choose the other parameter such that the value of the underlying assets agrees with that of the CAPM assets considered earlier.

We consider the Clayton copula as it is a simple one-parameter copula which exhibits lower tail dependence (Embrechts, Lindskog, and McNeil 2001). Again we choose the parameter so that the value of the underlying assets agrees with that of the CAPM assets $(\theta=0.659$ is the parameter thus chosen).

Application of Equation (12) produces the discount factors shown in Figure 3. Relative to the CAPM case (normal copula), the Student-t copula marginal SDF is of a similar shape in the middle of the distribution but applies a greater weight to the tails. Since the amount of debt has been chosen so that the likelihood of default is low, the region where loss occurs is reasonably far into the tail of the asset payoff distribution. The weighting applied by the Student-t SDF is thus significantly higher than that applied by the CAPM SDF in this region, and this is reflected in the credit spread for the debt, which in the Student-t case works out to be around 77 basis points, versus 60 basis points for the CAPM case.

The fact that the SDF in the Student-t case rises in the right-hand tail also is a consequence of the particular behaviour of the Student-t copula density, which approaches infinity in all four corners of the unit square - not just the high/high and low/low corners, but also the corner where the asset payoff is high and consumption is low. This means the corresponding marginal SDF puts a high weight on the very high end of the asset payoff as well as the low end. If that is not appropriate then some other copula should be assumed. For example, 


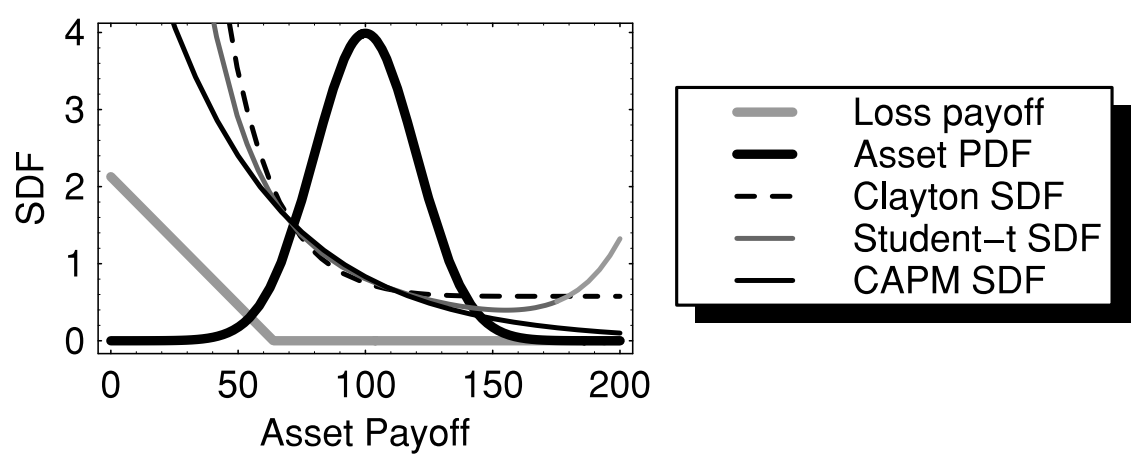

FIGURE 3: Asset payoff distribution, loss payoff, and stochastic discount factors, under three different assumptions about the copula between consumption and the underlying assets. All three SDF's are calibrated to give the same value for the underlying assets. Relative to the CAPM case (normal copula), the Student-t copula marginal SDF is of a similar shape in the middle of the distribution but applies a greater weight to the tails. The Clayton copula SDF applies even greater weight to the lower tail but is asymptotically horizontal in the upper tail.

the Clayton copula gives a marginal SDF which is horizontal in the upper tail and rises faster than that of the Student-t SDF in the lower tail. The credit spread turns out to be 95 basis points. The reason the Clayton copula SDF is horizontal in the upper tail is that in this upper tail consumption and the asset payoff are independent. By contrast, a derivative paying off further into the upper tail of the normal asset would attract a higher risk discount as the normal asset having a higher payoff implies consumption is likely to be higher. The exploration of the properties of the marginal SDF for a variety of copulas would be interesting, but we shall stop here for now, having exhibited the general pricing formula (12), and illustrated that changing the copula from normal can have a significant impact on asset prices.

\section{ConcLusions}

In this paper we have investigated the impact on asset prices of the dependence structure between the asset's payoff and aggregate consumption. We have performed this investigation in the context of an economy considered by Bühlmann and Wang, in which agents have exponential utility functions and aggregate consumption has a normal distribution. In this economy, the assets jointly normal with consumption satisfy the CAPM. Building on the work of Wang and Bühlmann we have given an explicit form for the marginal stochastic discount factor for this version of the CAPM.

We have then shown how to generalise the approach and calculate marginal stochastic discount factors for assets which have other marginal distributions and/or other copulas with consumption. We have shown that the choice of copula has a large impact on the prices of derivatives such as debt. In the example given above, the credit spread on debt increased by over $50 \%$ when 
moving from the normal copula to the Clayton copula, despite the underlying assets in each case having the same payoff distribution (cash flow) and the same value. Since there is no particular reason to believe that the normal copula assumption, which underlies the CAPM and the Black-Scholes formula in this setting, should in general be appropriate, there is a need to investigate what the appropriate copula is for various asset classes. This could be the subject of future empirical research.

Graphing the marginal discount factor provides useful insight into how derivatives of the asset will be valued. It is obvious from Figure 3 that out-ofthe-money call options written over the asset with normal copula will differ significantly in value from those written over the assets with Student-t or Clayton copulas, as the weightings applied in the upper tails will be quite different. It is accepted by practitioners that the Black-Scholes formula needs modification to deal with out-of-the-money options (the effect known as the "volatility smile"). The present paper shows that phenomena such as this could be explained by the copula between consumption and the underlying asset being other than normal, instead of or as well as the marginal distribution of the underlying asset being other than the log-normal assumed in Black-Scholes.

Other future research could include incorporation of the effects of real-world capital market imperfections, calibration to market data and subsequent comparison of model-implied debt prices to real debt prices. The methods employed here have broader applicability than debt pricing, of course; for example they could be used to value the liabilities of firms with risky claims, such as insurance firms, as well as for valuing equity options.

\section{REFERENCES}

Amato, J.D. and Remolona, E.M. (2003) The Credit Spread Puzzle, Bank of International Settlements Quarterly Review, 51-63. http://www.bis.org/publ/qtrpdf/r_qt0312e.pdf.

Black, F. and SchOles, M. (1972) The Valuation of Option Contracts and a Test of Market Efficiency, Journal of Finance 27(2), 397-418.

Black, F. and Scholes, M. (1973) The Pricing of Options and Corporate Liabilities, Journal of Political Economy 81(3), 637-654.

BühlmanN, H. (1980) An Economic Premium Principle, ASTIN Bulletin 11, 52-60.

Cochrane, J.H. (2001) Asset Pricing (Princeton University Press, Princeton, New Jersey).

Demarta, S. and McNeIL, A.J. (2004) The t Copula and Related Copulas, Working Paper, Department of Mathematics, ETHZ, www.math.ethz.ch/finance.

Embrechts, P., Lindskog, F. and McNeil, A. (2001) Modelling Dependence with Copulas and Applications to Risk Management, Working Paper, Department of Mathematics, ETHZ, www.math.ethz.ch/finance.

Leland, H.E. (1994) Corporate Debt Value, Bond Covenants, and Optimal Capital Structure, The Journal of Finance XLIX(4), 1213-1252.

Leland, H.E. and BJerRe Toft, K. (1996) Optimal Capital Structure, Endogenous Bankruptcy, and the Term Structure of Credit Spreads, The Journal of Finance LI(3), 987-1019.

LeRoy, S.F. and Werner, J. (2001) Principles of Financial Economics (Cambridge University Press, Cambridge, United Kingdom).

LinTNER, J. (1965) The Valuation of Risk Assets and the Selection of Risky Investments in Stock Portfolios and Capital Budgets, The Review of Economics and Statistics 47, 13-37. 
MCLeish, D.L. and ReESoR, R.M. (2003) Risk, Entropy and the Transformation of Distributions, North American Actuarial Journal 7(2).

Merton, R.C. (1974) On the Pricing of Corporate Debt: The Risk Structure of Interest Rates, The Journal of Finance 29(2), 449-470.

PANJER, H.H. et al. (1998) Financial Economics: with applications to investments, insurance and pensions (The Actuarial Foundation: Schaumburg, Illinois, USA).

SchÖnbucher, P.J. (2003) Credit Derivatives Pricing Models: Models, Pricing, Implementation (Wiley Finance).

Sharpe, W.F. (1964) Capital Asset Prices: A Theory of Market Equilibrium Under Conditions of Risk, The Journal of Finance XIX(3), 425-442.

Venter, G.G. (2001) Tails of Copulas, in Proceedings ASTIN Washington, USA, 68-113.

WANG, S.S. (2002) A Universal Framework for Pricing Financial and Insurance Risks, ASTIN Bulletin 32(2), 213-234.

WANG, S.S. (2003) Equilibrium pricing transforms: New results using Bühlmann's 1980 economic model, ASTIN Bulletin 33, 57-73.

MARK JOHNSTON

PricewaterhouseCoopers ${ }^{5}$

GPO Box 2650,

Sydney NSW 1171, Australia

and

University of New South Wales,

Sydney NSW 2052, Australia

E-mail:m.e.johnston@unsw.edu.au

5 Present address: Macquarie Bank, 1 Martin Place, Sydney NSW 2000, Australia. 